\title{
Papers
}

\section{Cost effectiveness of treatment for alcohol problems: findings of the randomised UK alcohol treatment trial (UKATT)}

\author{
UKATT Research Team
}

\begin{abstract}
Objective To compare the cost effectiveness of social behaviour and network therapy, a new treatment for alcohol problems, with that of the proved motivational enhancement therapy. Design Cost effectiveness analysis alongside a pragmatic randomised trial.

Setting Seven treatment sites around Birmingham, Cardiff, and Leeds.

Participants 742 clients with alcohol problems; 617 (83.2\%) were interviewed at 12 months and full economic data were obtained on 608 (98.5\% of 617$)$.

Main economic measures Quality adjusted life years (QALYs), costs of trial treatments, and consequences for public sector resources (health care, other alcohol treatment, social services, and criminal justice services).

Results Both therapies saved about five times as much in expenditure on health, social, and criminal justice services as they cost. Neither net savings nor cost effectiveness differed significantly between the therapies, despite the average cost of social behaviour and network therapy (£221; $\$ 385$; €320) being significantly more than that of motivational enhancement therapy (£129). If a QALY were worth $£ 30000$, then the motivational therapy would have $58 \%$ chance of being more cost effective than the social therapy, and the social therapy would have $42 \%$ chance of being more cost effective than the motivational therapy.

Conclusion Participants reported highly significant reductions in drinking and associated problems and costs. The novel social behaviour and network therapy did not differ significantly in cost effectiveness from the proved motivational enhancement therapy.
\end{abstract}

\section{Introduction}

Heavy drinking in England and Wales costs an estimated $£ 18000 \mathrm{~m}$ ( $\$ 32438 \mathrm{~m}$; €26 424m) each year as a result of costs for health and social care, losses to productivity, and criminal activity. ${ }^{1}$ Treatment for alcohol problems has the potential to improve the health of alcohol dependent individuals and to reduce social costs. Reviews of economic analyses of treatment for alcohol and other substance misuse have consistently found that health and other social costs decrease after treatment but have also identified methodological weaknesses. ${ }^{23}$ In particular, few have collected prospective data alongside randomised controlled trials.

Modelling of economic costs and consequences, mainly using expert opinions, and reviews of evidence suggested that brief motivational counselling, which includes motivational enhancement therapy, is more cost effective than many other types of treatment for alcohol problems. ${ }^{4}$ Modelling of long term health outcomes suggests that many psychosocial treatments, including motivational interviewing and training in social skills, reduce social costs. ${ }^{5}$

A large randomised trial of alcohol treatments in the United States (the "matching alcoholism treatments to client heterogeneity" trial) generated two economic analyses on completion. ${ }^{67}$ The first study modelled the costs of treatment from data on the uptake of therapies and calculated that motivational enhancement therapy would be less costly than cognitive behavioural therapy or twelve step facilitation in non-research settings. ${ }^{6}$ The second study analysed the health records of participants and concluded that healthcare costs decreased after treatment, confirming the economic benefits of treating alcohol problems. ${ }^{7}$

The UK alcohol treatment trial provided an opportunity to collect economic data alongside a large randomised trial. We collected data on general health outcomes, specific drinking outcomes, and a range of social effects. We designed the economic analysis to test whether social behaviour and network therapy, a new social treatment, was as cost effective in improving quality of life as motivational enhancement therapy, a motivational treatment of proved effectiveness. We compared the treatment costs, consequences for public sector resources, and health outcomes of the two therapies.

\section{Methods}

The UK alcohol treatment trial is described in detail elsewhere ${ }^{8}$ and summarised in the accompanying paper. ${ }^{9}$ Social behaviour and network therapy, comprising up to eight 50 minute sessions, helps clients to build social networks to support change in their drinking and associated behaviours. Motivational enhancement therapy, comprising up to three 50 minute sessions, combines counselling in the motivational style with objective individual feedback from earlier assessment.

We carried out our trial in seven UK sites around Birmingham, Cardiff, and Leeds. We trained 72 therapists in social behaviour and network therapy or motivational enhancement therapy through courses at Leeds lasting three days. To achieve accreditation the therapists had to show competence through video recordings and complete the supervised treatment of at least two clients in the motivational group or one client in the social network group (since clients in the social network group receive about twice as many sessions as those in the motivational group). Fifty two therapists achieved such accreditation. We recruited 742 clients with alcohol problems of all ages above 16 and from all social groups.

Full authorship details, the collaborators, and a detailed table of resources and costs are on bmj.com 
Table 1 Costs of public sector resources before and after randomisation by allocated treatment. Values are average numbers (SD) of units consumed unless stated otherwise

\begin{tabular}{|c|c|c|c|c|c|c|}
\hline \multirow[b]{2}{*}{ Resource } & \multirow[b]{2}{*}{ Unit } & \multirow[b]{2}{*}{$\begin{array}{l}\text { Cost }(£) \\
\text { per unit }\end{array}$} & \multicolumn{2}{|c|}{ Six months before randomisation } & \multicolumn{2}{|c|}{ Six months before follow-up } \\
\hline & & & $\begin{array}{l}\text { Motivational } \\
\text { group }(n=347)\end{array}$ & $\begin{array}{l}\text { Social network } \\
\text { group }(n=261)\end{array}$ & $\begin{array}{l}\text { Motivational } \\
\text { group }(n=347)\end{array}$ & $\begin{array}{l}\text { Social network } \\
\text { group }(n=261)\end{array}$ \\
\hline \multicolumn{7}{|l|}{ General health care ${ }^{10}$} \\
\hline Hospital inpatient & Night & 242 & $2.69(6.85)$ & $2.93(6.68)$ & $2.14(10.46)$ & $2.05(8.89)$ \\
\hline Hospital day patient & Visit & 77 & $0.04(0.20)$ & $0.02(0.16)$ & $0.03(0.17)$ & $0.03(0.16)$ \\
\hline Hospital outpatient & Appointment & 74 & $0.31(0.70)$ & $0.37(0.76)$ & $0.47(1.18)$ & $0.39(0.98)$ \\
\hline Hospital accident and emergency department & Visit & 61 & $0.55(0.83)$ & $0.50(0.81)$ & $0.28(0.61)$ & $0.33(0.71)$ \\
\hline \multicolumn{7}{|l|}{ General practitioner: } \\
\hline At home & Home visit & 59 & $0.12(0.45)$ & $0.15(0.46)$ & $0.12(0.65)$ & $0.12(0.98)$ \\
\hline In surgery & Consultation & 19 & $4.05(4.46)$ & $3.75(4.16)$ & $3.11(3.42)$ & $3.84(4.22)$ \\
\hline Prescriptions & Prescription & 19 & $11.61(18.00)$ & $12.39(17.90)$ & $11.65(19.80)$ & $11.68(21.90)$ \\
\hline Community psychiatric nurse & Home visit & 27 & $0.61(2.42)$ & $0.96(3.35)$ & $0.32(1.08)$ & $0.48(1.69)$ \\
\hline \multicolumn{7}{|l|}{ Specialist alcohol treatment } \\
\hline Detoxification in primary care ${ }^{2}$ & Episode & 350 & $0.16(0.71)$ & $0.18(0.66)$ & $0.06(0.47)$ & $0.04(0.39)$ \\
\hline \multicolumn{7}{|l|}{ Alcohol agency: } \\
\hline Rehabilitation $^{10}$ & Night & 89 & $0.32(4.17)$ & $0.24(2.28)$ & $0.13(0.97)$ & $0.10(0.98)$ \\
\hline Consultation* & Appointment & 28 & $1.83(2.56)$ & $1.85(2.37)$ & $1.03(9.90)$ & $1.33(7.61)$ \\
\hline Social services ${ }^{10}$ & Contact & 7 & $1.51(3.66)$ & $1.84(6.16)$ & $1.12(4.71)$ & $1.06(3.43)$ \\
\hline \multicolumn{7}{|l|}{ Criminal justice $^{11}$} \\
\hline \multicolumn{7}{|l|}{ Court attendance: } \\
\hline Crown court & Appearance & 8824 & $0.017(0.131)$ & $0.027(0.162)$ & $0.020(0.140)$ & $0.011(0.107)$ \\
\hline Magistrates court attendance & Appearance & 564 & $0.21(0.76)$ & $0.28(0.79)$ & $0.17(1.02)$ & $0.16(0.74)$ \\
\hline
\end{tabular}

${ }^{*}$ Estimated from data in UK alcohol treatment trial (excluding training costs).

We interviewed $617(83.2 \%)$ clients at 12 months. Of 352 clients in the motivational group, 347 (99\%) yielded data for economic analysis, together with $261(99 \%)$ of 265 clients in the social network group. We valued all economic costs and consequences in pounds sterling at 2000-1 prices. Discounting was not necessary as we measured costs and consequences occurring within a year of recruiting clients.

\section{Measurement of treatment costs}

We measured the time trainers and therapists spent in training and supervision, and their use of space and materials. We valued their time from their individual salaries, employers' costs, and overheads. ${ }^{10}$ We spread the total cost for training in each therapy over the corresponding number of sessions delivered for the UK alcohol treatment trial.

We measured the time therapists spent in delivering treatment from forms they completed after each session in each site, and we valued this time in the same way as the training costs. We measured the space and other resources therapists used at individual sites and valued these using local costs. To calculate the cost of treatment we multiplied session length in minutes by the cost per minute of the individual therapist and other resources. We derived the cost of treating each individual by summing over sessions.

\section{Measurement of public sector resource use}

We devised a questionnaire to measure, both at baseline and at 12 months, clients' use over the previous six months of health care, alcohol treatment outside our trial, social services, and the criminal justice system. We chose a period of six months so as to capture important but infrequent events within clients' recall. We estimated the unit costs of the 40 items covered by the questionnaire as long run marginal opportunity costs. Costs of health and social care items came from a national source, ${ }^{10}$ costs of crime came from government sources, ${ }^{11-13}$ and costs of alcohol treatment came partly from the UK alcohol treatment trial and partly from another UK study. ${ }^{5}$ Table 1 lists the more expensive resources, together with their average use, unit costs, and sources (see also table A on bmj.com).

\section{Measurement of health outcomes}

To permit comparison with other economic evaluations, we assessed health outcomes through quality adjusted life years (QALYs). ${ }^{14}{ }^{15}$ We asked participants to complete the EQ-5D questionnaire at baseline and at three and 12 months. ${ }^{16}$ This generic health status instrument measures health on five scales (mobility, self care, usual activities, pain and discomfort, and anxiety and depression) each with three points, thus putting participants into one of $243(=3 \times 3 \times 3 \times 3 \times 3)$ health states. We estimated how many QALYs participants had experienced during their year in the UK alcohol treatment trial by using UK population norms to value their health states ${ }^{17}$ and linear interpolation ${ }^{18}$ to calculate areas under the QALY curves. To impute missing final health states for a few respondents we carried forward their previous states.

\section{Economic and statistical analysis}

To improve estimates of QALYs we adjusted for differences in baseline measurements. Under specified assumptions analysis of covariance yields more precise estimates than analysis of changes between baseline and follow-up..$^{19}$ As the economic data collected in the UK alcohol treatment trial may violate several of these assumptions, however, it is acceptable and prudent to analyse changes between baseline and follow-up. ${ }^{20}$

As the economic data were skewed, we used bootstrapping to obtain more reliable confidence intervals. ${ }^{21}$ We used the statistical package STATA to draw 1000 artificial bootstrapped samples, each containing 608 observations drawn randomly with replacement from the actual sample of 608 participants with complete economic data. This enabled us to derive bootstrapped confidence intervals for key statistics, notably QALY gains and savings in public sector resources. We then divided the net cost of therapy by the QALYs gained to yield the average cost per QALY, otherwise known as the incremental cost effectiveness ratio. We estimated the sampling distribution of this ratio from the 1000 bootstrapped samples and derived cost effectiveness acceptability curves. These curves plot the resulting probability that one therapy is better than the other against the maximum that decision makers might pay for an additional QALY. ${ }^{21}$ 


\begin{tabular}{|c|c|c|c|}
\hline Variable & $\begin{array}{l}\text { Motivational group } \\
(\mathrm{n}=347)(£)\end{array}$ & $\begin{array}{l}\text { Social network group } \\
\quad(n=261)(£)\end{array}$ & $\begin{array}{l}\text { Difference } \\
(95 \% \mathrm{Cl})(£)\end{array}$ \\
\hline Basic assumptions & $129(58)$ & $221(179)$ & $92(69$ to 113$)$ \\
\hline \multicolumn{4}{|l|}{ Varying spread of training costs: } \\
\hline $\begin{array}{l}\text { Spread training costs over } 736 \text { treatment sessions that typical therapist delivers per year (16 sessions/week } \times 46 \\
\text { weeks) rather than sessions therapists delivered within UK alcohol treatment trial }\end{array}$ & $63(26)$ & $87(77)$ & 24 (15 to 32) \\
\hline \multicolumn{4}{|l|}{ Varying therapists' salaries: } \\
\hline$£ 20893$ (lower quartile) & $127(57)$ & $208(161)$ & 80 (62 to 99) \\
\hline £26 110 (higher quartile) & $132(59)$ & $216(169)$ & $83(65$ to 103$)$ \\
\hline \multicolumn{4}{|l|}{ Varying compliance with treatment (mean No of sessions): } \\
\hline Motivational sessions 1.89 (10th centile), social sessions 4.01 (90th centile) & $123(18)$ & $241(39)$ & 117 (112 to 122) \\
\hline Motivational sessions 2.12 (90th centile), social behaviour sessions 3.27 (10th centile) & $135(18)$ & $200(34)$ & $64(60$ to 69$)$ \\
\hline
\end{tabular}

\section{Sensitivity analyses}

To test whether differences in treatment costs between social behaviour and network therapy and motivational enhancement therapy depended on our assumptions, we undertook three distinct sensitivity analyses. We spread training costs over all 736 treatment sessions (16 sessions a week for 46 weeks) that a typical therapist might deliver in a year, rather than just the sessions therapists delivered within the UK alcohol treatment trial. We replaced the salaries of all 52 therapists in the UK alcohol treatment trial by the lower quartile of their annual salaries (£21 000 for a general counsellor) and then the higher quartile (£26 000 for a senior nurse). To test the effect of compliance with treatment we replaced the number of sessions attended for one treatment by the 10th centile of its distribution and the other by the 90th centile of its distribution, and vice versa.

\section{Results}

The 347 clients in the motivational group reported mean EQ-5D scores (health utilities) of 0.616 (SD 0.299) at baseline, 0.684 (SD 0.293 ) at three months, and 0.671 (SD 0.311) at 12 months. The corresponding values for the 261 clients in the social network group were 0.589 (SD 0.298), 0.648 (SD 0.314), and 0.626 (SD 0.324). After adjusting for baseline differences, we estimated that the social network group achieved 0.0113 fewer QALYs than the motivational group, but this was not significant (bias corrected 95\% confidence interval 0.0532 fewer to 0.0235 more).

\section{Treatment costs and resource savings}

The average treatment cost of social behaviour and network therapy (£221) was significantly greater than that of motivational enhancement therapy (£129; table 2 ). Three sensitivity analyses confirm the significantly higher cost of the social therapy (table 2). The difference ranged from $£ 24$ when spreading training costs over all 736 treatment sessions delivered by a typical therapist to $£ 117$ when comparing the 10th centile of the number of sessions for the motivational therapy (1.9) with the 90th centile of the number of sessions for the social therapy (4.0).

In the six months before randomisation, $590(97.0 \%)$ of 608 participants had used health care and $572(94.1 \%)$ had been in contact with services providing treatment for alcohol problems, but only $286(47.0 \%)$ had used social services and only 128 (21.1\%) had had any contact with criminal justice. In the six months before follow-up all these percentages fell: 517 (85.0\%) used health care, only $164(27.0 \%)$ contacted alcohol treatment services, $170(28.0 \%)$ used social services, and $61(10.0 \%)$ had any contact with criminal justice. Valuing these differences shows that both treatments resulted in substantial and similar savings in public sector resources-namely, about five times as much as they cost (table 3). Combining treatment costs with these savings suggests that social behaviour and network therapy achieved a mean net public sector saving of $£ 206$ per client more than motivational enhancement therapy, but the difference was not significant (95\% confidence interval - £454 to £818).

Table 3 Costs of public sector resources at 2000-1 prices by allocated treatment. Values are mean (standard deviation) costs of alcohol problems unless stated otherwise

\begin{tabular}{|c|c|c|c|}
\hline Public sector resource costs & $\begin{array}{l}\text { Motivational group } \\
\quad(\mathrm{n}=347)\end{array}$ & $\begin{array}{l}\text { Social network group } \\
\qquad(\mathrm{n}=261)\end{array}$ & $\begin{array}{l}\text { Difference } \\
(95 \% \mathrm{Cl}) \dagger\end{array}$ \\
\hline \multicolumn{4}{|l|}{ Six months before randomisation: } \\
\hline Health care & 1121 (1953) & $1192(1829)$ & $71(-219$ to 389$)$ \\
\hline Criminal justice & $519(1524)$ & $685(1823)$ & $166(-104$ to 466$)$ \\
\hline Other alcohol treatment & $502(1456)$ & $621(1071)$ & 119 (-103 to 299) \\
\hline Social care & $50(133)$ & $87(303)$ & $37(3 \text { to } 84)^{\star}$ \\
\hline Total & $2192(3409)$ & $2585(3224)$ & 393 (-118 to 918$)$ \\
\hline \multicolumn{4}{|l|}{ Six months before follow-up: } \\
\hline Health care & $900(2693)$ & $912(2330)$ & $13(-419$ to 379$)$ \\
\hline Criminal justice & $351(1851)$ & $301(1241)$ & $-50(-315$ to 181$)$ \\
\hline Other alcohol treatment & $186(806)$ & $290(1037)$ & 104 (-31 to 271) \\
\hline Social care & $34(107)$ & $63(208)$ & $29(3 \text { to } 58)^{*}$ \\
\hline Total & $1469(3466)$ & $1565(3171)$ & 96 (-435 to 602) \\
\hline Reduction in public sector resources: 6 months before randomisation versus 6 months before follow-up & $722(4116)$ & $1020(3802)$ & 298 (-353 to 909) \\
\hline Cost of specialist alcohol treatment within trial & $129(58)$ & $221(178)$ & $92(69 \text { to } 113)^{\star *}$ \\
\hline Net reduction in public sector resource costs minus cost of trial treatments & $593(4114)$ & 798 (3817) & $206(-454$ to 818$)$ \\
\hline
\end{tabular}

*Statistically significant difference between treatments at $5 \%$ level.

†Confidence interval corrected for bias.

*** Statistically significant difference between treatments at $0.1 \%$ level. 


\section{Cost utility analysis}

Combining the net savings from social behaviour and network therapy with the net health utility gains it achieved yields an incremental cost effectiveness ratio which is the ratio of two negative but non-significant quantities. To summarise this double negative, motivational enhancement therapy has an incremental cost effectiveness ratio of $£ 18230$ (marginal cost of $£ 206$ divided by marginal utility gain of 0.0113 ) relative to the social therapy.

Bootstrapping generated 1000 different artificial samples to characterise the distribution of health outcomes and net costs (treatment costs less public sector savings) between the two treatments. In 233 samples social behaviour and network therapy was more effective than motivational enhancement therapy in generating QALYs and less costly in net public sector resources (social therapy dominated motivational therapy). In 193 the social therapy was more costly and less effective than the motivational therapy (motivational therapy dominated social therapy). In 514 the social therapy was less costly but less effective than the motivational therapy, and in the remaining 60 the social therapy was more effective but more costly than the motivational therapy. Thus $19 \%$ of bootstrapped samples unequivocally favoured motivational enhancement therapy whereas $23 \%$ unequivocally favoured social behaviour and network therapy-a difference of only $4 \%$.

Plotting the resulting probability that the motivational therapy is more cost effective than the social therapy against the maximum that decision makers might pay for a QALY shows that the two therapies were similarly cost effective (figure). When this threshold is small, the social therapy seems preferable to the motivational therapy. For example, when a QALY is worth nothing to decision makers, the social therapy would be preferable to the motivational therapy in $747(74.7 \%)$ of the 1000 samples (514 where the health gain from motivational therapy then has no value, plus 233 where social therapy dominates). As the threshold increases, the motivational therapy becomes more attractive because the possible health gains from the motivational therapy are more highly valued than the possible cost savings from the social therapy. When a QALY is worth $£ 100000$ to decision makers, the motivational therapy is preferable to the social network therapy in $662(66.2 \%)$ of the 1000 samples). Between these two points, if decision makers value an additional QALY at $£ 30000$ (a value consistent with previous recommendations made by the National Institute for Clinical Excellence in the United Kingdom), ${ }^{22}$ then motivational enhancement therapy has a $57.6 \%$ probability of being more cost effective than social behaviour and network therapy, and the complementary probability for social behaviour and network therapy is $42.4 \%$.

\section{Discussion}

In the accompanying clinical paper on the UK alcohol treatment trial, we concluded that social behaviour and network therapy (a novel treatment for alcohol problems) was as effective as motivational enhancement therapy (a treatment of proven effectiveness). ${ }^{9}$ Participants reported highly significant improvements in drinking and associated problems, and in mental health. Our economic analysis alongside the UK alcohol treatment trial found no evidence of differences in net cost. Although the direct cost of the social therapy was $£ 92$ per client more than that of the motivational therapy, net savings in public sector resources, including those devoted to health and social care and to criminal justice, did not differ significantly between the two therapiesboth saved about five times as much as they cost.

We have carried out the first prospective cost effectiveness analysis in alcohol research alongside a large multicentre pragmatic randomised trial (the UK alcohol treatment trial). In this analysis we estimated health gains and clients' use of 40 different public services. Nevertheless we did not include loss of productivity or measure the full social costs of either alcohol related violence or the effects of alcohol problems on family or friends; in particular we could not tell whether effects on family and friends were increased or reduced by social behaviour and network therapy. There was some evidence that using either therapy increased time in employment. If we had not avoided adding gains in productivity to gains in quality of life within economic analysis, however, we would have counted benefits twice. ${ }^{14}$

The UK alcohol treatment trial reinforces the finding that treatment for alcohol problems leads to net savings. ${ }^{23}{ }^{24}$ We estimated these savings by comparing clients' use of services during the six months before randomisation with their use during the same period in the following year. If use of services before entering treatment is higher than normal, this technique may overestimate savings. In contrast, if the therapies each generate savings during treatment ${ }^{25}$ or after 12 months, this technique may underestimate savings. We did not attempt to model the long term effects of reduced drinking on health.

In the United Kingdom the National Institute for Clinical Excellence seems to value an additional quality adjusted life year (QALY) between $£ 20000^{15}$ and $£ 30000 .^{22}$ Even if the value of a QALY were $£ 30000$, motivational enhancement therapy would have $58 \%$ chance of being more cost effective than social behaviour and network therapy; by subtraction the social therapy would have $42 \%$ chance of being more cost effective than the motivational therapy. Participants allocated at random to the reference group received motivational enhancement therapy, a treatment of proved effectiveness. Therefore the combination of a sample size large enough to detect small differences and the lack of significant differences leads to positive conclusions about the value of both of these treatments for alcohol problems. In particular we judge that our confidence intervals for differences in utility and differences in cost exclude differences between the therapies that would have led to unequivocal recommendations in favour of one treatment.

In short we found evidence that social behaviour and network therapy is as effective as motivational enhancement therapy, and that both are cost effective. Thus commissioners of treatment for alcohol problems are free to choose either or both

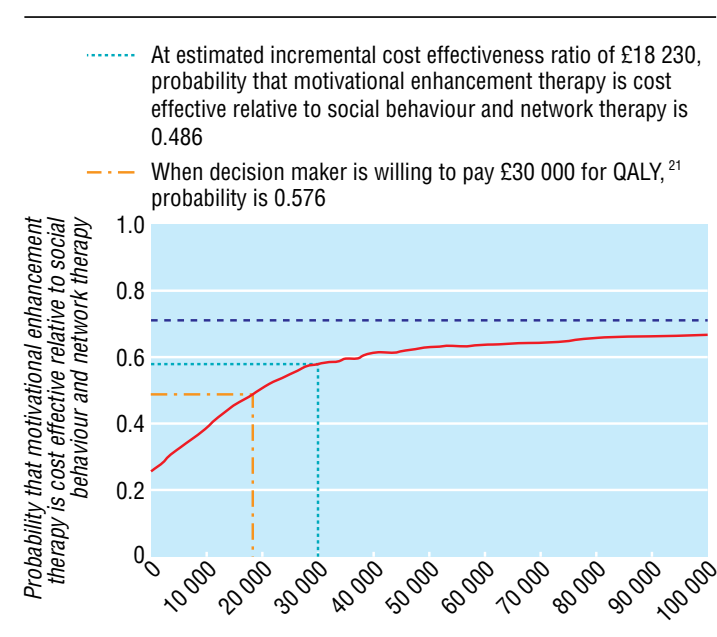

Maximum that decision maker is willing to pay for extra $Q A L Y$

Cost effectiveness acceptability curve for motivational enhancement therapy relative to social behaviour and network therapy 


\section{What is already known on this topic}

Studies suggest that psychosocial treatment for alcohol problems is cost effective, yet many have methodological weaknesses

\section{What this study adds}

Social behaviour and network therapy did not differ significantly in cost effectiveness from motivational enhancement therapy

Both therapy groups reported substantially reduced drinking and associated problems and achieved large savings in the costs of health and social care and of criminal justice

treatments on the basis of local circumstances, notably the availability of therapists of each type in statutory and non-statutory services. Although both treatments give value for money, both need trained therapists. We found that training is feasible and cheap. Hence there is now strong evidence about the effectiveness and cost effectiveness of two complementary treatments for alcohol problems.

Continuing analysis of the UK alcohol treatment trial data will focus on the crucial question whether we can characterise who respond better to motivational enhancement therapy than to social behaviour and network therapy and vice versa, and thus achieve even greater benefits from each treatment at no additional cost.

We thank the participants for their contributions.

The Medical Research Council established a trial steering committee to monitor the progress of the trial.

Contributors: AC was responsible for research and clinical management in the West Midlands. He contributed to the original grant application, the design, implementation, and management of the trial, analysing and interpreting data, and writing the paper. SC was responsible for designing and implementing the data management plan, and scanning, verifying, and validating data. He contributed to implementing the trial design, developing and implementing randomisation procedures, developing questionnaires and data collection instruments, analysing and interpreting data, and redrafting the paper. $\mathrm{AF}$ was responsible for designing and implementing the analysis plan, and validating data statistically. She contributed to implementing the trial design, developing and implementing randomisation procedures, developing data collection instruments, analysing and interpreting data, and redrafting the paper. CG was responsible for health economics. She contributed to the original grant application, the design, management, and implementation of the trial, analysing and interpreting data, and writing the paper. $\mathrm{NH}$ was responsible for research coordination. He contributed to the original grant application and participated in the design, implementation, and management of the trial, managing, analysing, and interpreting data, and writing the paper. RH was responsible for research and clinical management in South Wales. He contributed to the original grant application, the design, implementation and management of the trial, analysing and interpreting data, and writing the paper. VM contributed to validating, analysing, and interpreting data, and writing the paper. JO was responsible for trial coordination. He contributed to the original grant application, the design, implementation, and management of the trial, managing, analysing, and interpreting data, and writing the paper. SP was responsible for designing and implementing the health economic assessment. He contributed to implementing the trial design, developing procedures, questionnaires, and other instruments, analysing and interpreting data, and writing the paper. DR was responsible for research and clinical management in Leeds. He contributed to the original grant application, the design, implementation and management of the trial, analysing and interpreting data, and writing the paper. IR was responsible for trial methods. He contributed to the original grant application, the design, implementation, and management of the trial, managing, analysing, and interpreting data, and writing the paper. DR and IR act as guarantors. GS was responsible for designing and implementing trial management and quality assurance procedures, and coordinating the work of the treatment sites. He contributed to the implementation and management of the trial, developing procedures, questionnaires, and other instruments, designing and implementing the data management plan, verifying, validating, analysing, and interpreting data, and redrafting the paper. $\mathrm{G}$ Tober was responsible for therapist training and supervision. She contributed to the original grant application, the design, implementation, and management of the trial, the management and analysis of video recordings of therapy within the trial, analysing and interpreting data, and writing the paper. The local research coordinators responsible for implementing and managing the trial were: $\mathrm{G}$ Thistlethwaite in the integrated treatment site in Leeds; TA across four treatment sites in South Wales; and CK across two treatment sites in the West Midlands. The main follow-up interviewers were: LH and AW in Leeds; RB and BJ in South Wales; and KF and MS in the West Midlands. RB and BJ later became local research coordinators in succession to TA, and MS in succession to CK. At the trial training centre in Leeds, OF and WM analysed the video recordings of therapy. These fieldworkers also contributed to developing procedures, questionnaires, and other instruments. Together with other authors they commented on successive drafts of the paper.

Funding: Medical Research Council, National Health Service Executive in England, and Wales Office for Research and Development in Health and Social Care.

Competing interests: None declared.

Ethical approval: Relevant local research ethics committees approved the trial protocol.

1 Leontaridi R. Alcohol misuse: how much does it cost? London: Cabinet Office, 2003. www.strategy.gov.uk/files/pdf/econ.pdf (accessed 25 April 2005).

2 Ludbrook A, Godfrey C, Wyness L, Parrott S, Haw S, Napper M, et al. Effective and costeffective measures to reduce alcohol misuse in Scotland. Edinburgh: Scottish Executive Health Department, 2001.

3 McCollister KE, French MT. The relative contribution of outcome domains in the total economic benefit of addiction interventions: a review of first findings. Addiction 2003;98:1647-59.

4 Holder HD, Longabaugh R, Miller WR, Rubonis AV. The cost effectiveness of treatment for alcoholism: a first approximation.J Stud Alcohol 1991;52:517-40.

5 Slattery J, Chick J, Cochrane M, Craig J, Godfrey C, Kohli H, et al. Prevention of relapse in alcohol dependence. Health technology assessment report 3. Glasgow: Health Technology Board for Scotland, 2003.

6 Cisler R, Holder HD, Longabaugh R, Stout RL, Zweben A. Actual and estimated replication costs for alcohol treatment modalities: case study from project MATCH. J Stud Alcohol 1998;50:503-12.

7 Holder HD, Cisler R, Longabaugh R, Stout RL, Treno AJ, Zweben A. Alcoholism treatment and medical care costs from project MATCH. Addiction 2000;95:999-1014.

8 UKATT Research Team. United Kingdom alcohol treatment trial (UKATT): hypotheses, design and methods. Alcohol Alcohol 2001;36:11-21.

9 UKATT Research Team. Effectiveness of treatment for alcohol problems: findings of UKATT Research Team. Effectiveness of treatment for alcohol problems:
the randomised UK alcohol treatment trial (UKATT). BMJ 2005;331:541-4.

10 Netten A, Dennett J, Knight J. Unit costs of health and social care. Canterbury: Personal Social Services Research Unit, University of Kent, 2002.

11 Harries R. The cost of criminal justice. Home Office research findings 103. London: Home Office, 1999.

12 Home Office. Statistics on race and the criminal justice system. London: Home Office, 1998. 13 HM Treasury. Public expenditure statistical analysis 2000-1. Command 4601. London: Stationery Office, 2000.

14 Gold MR, Siegel JE, Russell LB, Weinstein MC. Cost-effectiveness in health and medicine. New York: Oxford University Press, 1996.

15 National Institute for Clinical Excellence. Guide to the methods of technology appraisal. London: National Institute for Clinical Excellence, 2004.

16 Brooks R. EuroQol: the current state of play. Health Policy 1996;37:53-72.

17 Kind P, Hardman G, Macran S. UK population norms for EQ-5D. Discussion paper 172. York: Centre for Health Economics, University of York, 1999.

18 Richardson G, Manca A. Calculation of quality adjusted life years in the published literature: a review of methodology and transparency. Health Econ 2004;13:1203-10.

19 Vickers AJ, Altman DC. Analysing controlled trials with baseline and follow up measurements. BMJ 2001:323:1123-4.

20 Vickers AJ. The use of percentage change from baseline as an outcome in a controlled trial is statistically inefficient: a simulation study. BMC Med Res Methodol 2001;1-6.

21 Briggs AH, Gray AM. Handling uncertainty when performing economic evaluation of Briggs AH, Gray AM. Handling uncertainty when perfor
health care interventions. Health Technol Assess 1999;3(2).

22 Raftery J. NICE-faster access to modern treatments? Analysis of guidance on health technologies. BMJ 2001;323:1300-3.

23 Potamianos G, North W, Meade T, Townsend J, Peters T. Randomised trial of community-based versus conventional hospital management in the treatment of alcoholism. Lancet 1986;ii:797-9.

24 Parthasarathy S, Weisner C, Hu T-W, Moore C. Association of outpatient alcohol and drug treatment with health care utilization and cost: revisiting the offset hypothesis. J Stud Alcohol 2001;62:89-97.

25 Gerstein DR, Johnson RA, Harwood HJ, Fountain K, Suter N, Malloy K. Evaluating recovery services: the California drug and alcohol treatment assessment (CalDATA) general report. Sacramento, CA: California Department of Alcohol and Drug Progams, 1994.

bmj.com 2005;331:544

See web extra for full authorship details

Correspondence to: Christine Godfrey, professor, Department of Health Sciences, University of York, YO10 5DD cg2@york.ac.uk 